\title{
Subsyndromal delirium in old age: conceptual and methodological issues
}

Delirium is a cognitive disorder characterized by acute onset, fluctuating course, altered level of consciousness, inattention, disorganized thinking, disorientation, memory impairment, and perceptual and motor disturbances (American Psychiatric Association, 2000; World Health Organization, 2010). It occurs in hyperactive, hypoactive, or mixed forms in up to $42 \%$ of older hospital inpatients (Siddiqi et al., 2006) and 70\% of older long-term care residents (McCusker et al., 2011). In both settings, delirium is independently associated with poor outcomes (Siddiqi et al., 2006; McCusker et al., 2010; Witlox et al., 2010).

Both DSM-IV-TR and ICD-10 diagnostic criteria for delirium require the coexistence of symptoms from multiple domains (American Psychiatric Association, 2000; World Health Organization, 2010). It is common, however, for older people to display one or more symptoms of delirium without having the full syndrome (Rockwood, 1993; Kiely et al., 2003). The occurrence of such symptoms has been labeled as subsyndromal delirium (SSD) (Levkoff et al., 1996).

The existence of SSD is controversial. Some investigators claim that SSD may be a clinically important condition that falls on a continuum between no symptoms and full delirium, perhaps a marker for underlying medical conditions (e.g. infection, drug toxicity) not severe enough to cause full delirium (Levkoff et al., 1996; Cole et al., 2003). Others argue that distinguishing subsyndromal presentations from full delirium is not "clinically practical given the fluctuating course of delirium, as clinical manifestations may range from normality through subsyndromal to full syndromal delirium in a matter of hours" (Blazer and van Nieuwenhuizen, 2012).

To date, evidence for the existence of SSD is inconclusive. On the one hand, two studies of the course of SSD have reported that episodes of SSD appear to occur independently of full delirium, last for 1-133 days, and often end in recovery but often recur (Tan et al., 2008; Cole et al., 2012a). On the other hand, a systematic review of the studies of SSD has reported significant unexplained heterogeneity in the results of studies of prevalence, incidence, and some risk factors that may undermine the credibility of the findings of these studies (Cole et al., 2012b).
Only further research will advance knowledge and determine whether or not SSD is a clinically important condition. To advance knowledge, such research must wrestle with six conceptual and methodological issues and try to avoid teleological reasoning and research design. These issues include the following: the types of symptoms of delirium required for the diagnosis of SSD, the number of symptoms required for diagnosis, the relationship of the symptoms present to full delirium, the measurement of the symptoms of delirium, prevalent versus incident SSD, and the selection of patient populations. Each of these issues is outlined below.

\section{Types of symptoms of delirium}

To date, three studies (Levkoff et al., 1996; Cole et al., 2003; Liptzin et al., 2005) have defined SSD as the presence of one or more symptoms of delirium; the symptoms were inattention, altered level of consciousness, disorientation, and perceptual disturbances. Five studies (Marcantonio et al., 2002; Bourdel-Marchasson et al., 2004; Tan et al., 2008; Leonard et al., 2009; Cole et al., 2011) have defined SSD as the presence of one or more Confusion Assessment Method (CAM) core symptoms of delirium; the CAM core symptoms were acute onset and fluctuation, inattention, disorganized thinking, and altered level of consciousness. Three studies (Ouimet et al., 2007; Ceriana et al., 2010; Skrobik et al., 2010) have defined SSD as the presence of one to three symptoms of delirium on the Intensive Care Delirium Screening Checklist; the symptoms included altered level of consciousness, inattention, disorientation, hallucinations or delusions, agitation or retardation, inappropriate speech or mood, sleep/wake cycle disturbance, and symptom fluctuation. One study (Meagher et al., 2012) has defined SSD as a severity score of 8-15 on the 13-item severity scale of the Delirium Rating Scale-98 (DRS-98); the severity scale includes disturbances in sleep-wake cycle, perception, thought form and content, affect, language, motor activity, orientation, attention, short- and long-term memory, and visuospatial ability. Finally, one study (Trzepacz et al., 2012) has defined SSD 
using a type of cluster analysis of the 13 severity items and two diagnostic items of the DRS-98. SSD was intermediate in severity between no symptoms and full delirium, but its phenotype was more like delirium; proposed criteria for SSD include acute change from baseline, thought disorder, disorientation to time and place and mild disturbances in sleep-wake cycle, inattention, and navigating in one's surroundings.

Of note, differences in the prevalence and incidence of SSD cannot be attributed to the diagnostic criteria used (Cole et al., 2012b). Only one study has compared prevalence rates of SSD using two different sets of diagnostic criteria and reported little difference in the rates (Voyer et al., 2009). The reason for the above findings may be that, despite differences in diagnostic criteria between studies, the symptoms that most frequently identify SSD are the same in all studies: to wit, two studies report that SSD was identified most often by the symptom of inattention (Cole et al., 2003, 2011) and this symptom is common to all sets of diagnostic criteria.

\section{Number of symptoms of delirium}

Most studies of SSD have defined SSD by the presence of one or more symptoms of delirium, or two or more symptoms; consequently, it is difficult to evaluate the frequency or significance of the presence of one versus two versus three symptoms. That being said, three studies have compared the frequency of SSD defined by the presence of one versus two to three Confusion Assessment Method (CAM) core symptoms of delirium (Kiely et al., 2003; Dosa et al., 2007; von Gunten and Mosimann, 2010); and one study has compared the frequency and outcomes of SSD defined as the presence of one or more versus two or more CAM core symptoms (Cole et al., 2011). Although the evidence is limited, it appears that the more the symptoms required for SSD diagnosis, the lower the prevalence and incidence rates and the poorer the outcomes.

\section{Relationship of symptoms present to delirium}

When SSD was described by Guainerio in 1517 (Diethelm, 1971), “... he emphasized that a predelirious period ... can be recognized which ... may not lead to full delirium." Similarly, Lipowski (1990) described a "prodromal phase ... in which patients had one or more symptoms of delirium (decreased concentration and ability to think, restlessness, anxiety, irritability, drowsiness, hypersensitivity to stimuli, nightmares) that never progressed to full DSM-defined delirium."

Many authors, however, have not distinguished between symptoms that do or do not progress to delirium (Kiely et al., 2003; Marcantonio et al., 2005; Dosa et al., 2007; Voyer et al., 2009; von Gunten and Mosimann, 2010). DSM-IV-TR recognizes "subsyndromal presentations ... with some but not all of the symptoms of delirium" and recommends that such presentations be coded as Cognitive Disorder Not Otherwise Specified (American Psychiatric Association, 2000). More recently, the DSM-V Neurocognitive Disorders Workgroup has discussed whether to add SSD as a subcategory of delirium in parallel with a new category, mild neurocognitive disorder (Jeste, 2010). Notably, neither DSM-IV-TR nor the DSM-V Workgroup distinguishes between subsyndromal presentations that do or do not progress to delirium.

Whether or not SSD occurs independently of delirium is a critical issue for research. Many symptoms of delirium appear to occur before and after episodes of delirium, at least in some populations (Cole et al., 2012c). If the definition of SSD does not exclude symptoms that occur immediately before or after episodes of delirium, it may not be possible to distinguish the risk factors, course, and outcomes of SSD from those of delirium. If the definition of SSD excludes symptoms that occur immediately before or after episodes of delirium, it may be possible to distinguish the risk factors, course, and outcomes of SSD from those of delirium and investigate the possibility of a continuum of acute neurocognitive disorder in older people.

Of note, in 12 studies that excluded SSD progressing to delirium, the combined prevalence of SSD was $23 \%(95 \%$ CI $=9 \%-42 \%)$; the combined incidence was $13 \%(95 \% \mathrm{CI}=6 \%-$ $23 \%)$; risk factors were older age, dementia, more cognitive and BADL impairment, admitted from an institution, increasing severity of medical illness, vision impairment, and more co-morbidity; outcomes (i.e. cognitive and functional decline, increased length of hospital stay and increased rates of admission to long-term care institutions, and death) were poor (Cole et al., 2012b). In five studies that did not exclude SSD progressing to delirium, the reported prevalence of SSD ranged from $11.9 \%$ to $51 \%$; the median rate was $39.5 \%$. Two of these five studies reported risk factors similar to SSD not progressing to delirium and one reported outcomes similar to SSD not progressing to delirium. Notably, none of the five studies reported the proportions of subjects with SSD that did or did not progress to full delirium. Although the evidence is limited, it appears that the inclusion of subjects with SSD 
that may progress to delirium increases prevalence substantially but may not change risk factors or outcomes.

\section{Measurement of symptoms of delirium}

To date, the following four different instruments have been used to identify SSD: the Delirium Symptom Interview (Albert et al., 1992), the Confusion Assessment Method (Inouye et al., 1990), the Intensive Care Delirium Screening Checklist (Bergeron et al., 2001), and the Delirium Rating Scale-98 (Trzepacz et al., 2001). There do not appear to be consistent differences in the frequencies, risk factors, or outcomes of SSD when these different instruments are used (Cole et al., $2012 \mathrm{~b}$ ), perhaps because a relatively small set of symptoms (e.g. inattention, disorganized thinking) identify most cases of SSD and are common to most of the instruments. Although the evidence is limited, the instrument(s) that best measure the small set of symptoms relevant to the detection of SSD are likely to be the most useful.

As yet, there is little consensus on the duration or frequency of measurement required to detect SSD. In published studies, the period of observation ranges from 5-180 days, median 7-8 days; when the period of observation has involved more than one assessment, the frequency of assessments has ranged from thrice per day to weekly, median daily (Cole et al., 2012b).

\section{Prevalent versus incident SSD}

Prevalent SSD can be defined as a diagnosis of SSD at the time of first assessment. Incident SSD can be defined as a diagnosis of SSD following one or more assessments with no symptoms of delirium. Because prevalent SSD may represent the end of a resolving episode of delirium or even persistent symptoms of delirium (Cole, 2010), inclusion of prevalent cases in studies of SSD may preclude the study of SSD, independent of delirium.

\section{Selection of patient populations}

The prevalence and incidence of delirium can vary enormously by type of population selected (e.g. community, emergency room, medical inpatients, surgical inpatients, nursing home residents) and the frequency of dementia in the population selected (McCusker et al., 2011). Risk factors for delirium tend to be similar among medical and surgical populations but may be different among nursing home populations (McCusker et al., 2011). Outcomes seem to be uniformly poor across all populations studied, perhaps worse among those with dementia (McCusker et al., 2001). Because findings will probably be similar for SSD, it may be important to consider the populations selected to study SSD.

\section{Conclusion}

What is SSD? What is the clinical significance of SSD? Does SSD occur independently of delirium? Is SSD a marker for underlying medical conditions not severe enough to cause delirium? Is SSD a clinically important condition requiring medical attention? Or does SSD reflect only the fluctuating nature of delirium and the fact that infrequent assessments of symptoms of delirium miss the diagnosis of full delirium? To answer these questions, further research must grapple with six conceptual and methodological issues and try to avoid teleological reasoning and research design. The issues include the following: the types and number of symptoms of delirium required for the diagnosis of SSD, the relationship of the symptoms present to full delirium, the measurement of the symptoms of delirium, enrolment of prevalent versus incident SSD, and the selection of patient populations. Research that satisfactorily addresses these issues may advance knowledge of SSD.

\section{Conflict of interest}

None.

\section{Martin G. Cole}

Department of Psychiatry, St Mary's Hospital and McGill University Montreal, Quebec, Canada

Email: martin.cole@ssss.gouv.qc.ca

\section{References}

Albert, M. S. et al. (1992). The Delirium Symptom Interview: an interview for the detection of delirium symptoms in hospitalized patients. Fournal of Geriatric Psychiatry and Neurology, 5, 14-21.

American Psychiatric Association (2000). Diagnostic and Statistical Manual of Mental Disorders, 4th Text Revision (DSM-IV-TR). Washington, DC: American Psychiatric Association.

Bergeron, N., Dubois, M.-J., Dumont, M., Dial, S. and Skrobik, Y. (2001). Intensive care delirium screening checklist: evaluation of a new screening tool. Intensive Care Medicine, 27, 859-864.

Blazer, D. G. and van Nieuwenhuizen, A. O. (2012). Evidence for the diagnostic criteria of delirium: an update. Current Opinion in Psychiatry, 25, 239-243.

Bourdel-Marchasson, I. et al. (2004). Delirium symptoms and low dietary intake in older inpatients are independent 
predictors of institutionalization: a 1-year prospective population-based study. Fournals of Gerontology: Series $A$ Biological Sciences and Medical Sciences, 59A, 350-354.

Ceriana, P., Fanfulla, F., Mazzacane, F., Santoro, C. and Nava, S. (2010). Delirium in patients admitted to a step-down unit: analysis of incidence and risk factors. Fournal of Critical Care, 25, 136-143.

Cole, M. G. (2010). Persistent delirium in older hospital patients. Current Opinion in Psychiatry, 23, 250-254.

Cole, M., McCusker, J., Dendukuri, N. and Han, L. (2003). The prognostic significance of subsyndromal delirium in elderly medical inpatients. Fournal of the American Geriatrics Society, 51, 754-760.

Cole, M. G. et al. (2011). Subsyndromal delirium in older long-term care residents: incidence, risk factors, and outcomes. Fournal of the American Geriatrics Society, 59, 1829-1836.

Cole, M. G. et al. (2013). The course of subsyndromal delirium in older long-term care residents. The American Fournal of Geriatric Psychiatry, 21, 289-296.

Cole, M. G., Ciampi, A., Belzile, E. and Dubuc-Sarrasin, M. (2012b). Subsyndromal delirium in older people: a systematic review of frequency, risk factors, course and outcomes. International fournal of Geriatric Psychiatry, doi:10.1002/gps.3891.

Cole, M. G. et al. (2012c). Symptoms of delirium occurring before and after episodes of delirium in older long-term care residents. Fournal of the American Geriatrics Society, 60, 2302-2307.

Diethelm, O. (1971). Medical Dissertations of Psychiatric Interest, Printed Before 1750. Basel, Switzerland: S. Karger.

Dosa, D., Intrator, O., McNicoll, L., Cang, Y. and Teno, J. (2007). Preliminary derivation of a nursing home confusion assessment method based on data from the minimum data set. Fournal of the American Geriatrics Society, 55, 1099-1105.

Inouye, S. K. et al. (1990). Clarifying confusion: the Confusion Assessment Method, a new method for detection of delirium. Annals of Internal Medicine, 113, 941-948.

Jeste, D. (2010). DSM-V Neurocognitive Criteria, Draft 1/7/10. Available at:http://www.dsm5.org/Proposed\%20Revision\% 20Attachments/APA\%20Neurocognitive \%20Disorders \% 20Proposal\%20for\%20DSM-5.pdf; last accessed 25 February 2013.

Kiely, D. K. et al. (2003). Delirium among newly admitted postacute facility patients: prevalence, symptoms, and severity. Fournals of Gerontology: Series A Biological Sciences and Medical Sciences, 58, M441-M445.

Leonard, M. et al. (2009). Symptoms of depression and delirium assessed serially in palliative-care inpatients. Psychosomatics, 50, 506.

Levkoff, S. E. et al. (1996). Subsyndromal delirium. American Fournal of Geriatric Psychiatry, 4, 320-329.

Lipowski, Z. J. (1990). Delirium: acute confusional states. In Z. J. Lipowski (ed.), Clinical Features, Course, and Outcome (pp. 54-57). Oxford, UK: Oxford Universiy Press.

Liptzin, B., Laki, A., Garb, J. L., Fingeroth, R. and Krushell, R. (2005). Donepezil in the prevention and treatment of post-surgical delirium. American fournal of Geriatric Psychiatry, 13, 1100-1106.
Marcantonio, E., Ta, T., Duthie, E. and Resnick, N. M. (2002). Delirium severity and psychomotor types: their relationship with outcomes after hip fracture repair. Fournal of the American Geriatrics Society, 50, 850-857.

Marcantonio, E. R. et al. (2005). Outcomes of older people admitted to postacute facilities with delirium. Fournal of the American Geriatrics Society, 53, 963-969.

McCusker, J., Cole, M., Dendukuri, N., Belzile, E. and Primeau, F. (2001). Delirium in older medical inpatients and subsequent cognitive and functional status: a prospective study. Canadian Medical Association fournal, 165, 575-583.

McCusker, J. et al. (2010). Use of nurse-observed symptoms of delirium in long-term care: effects on prevalence and outcomes of delirium. International Psychogeriatrics, 23, 602-608.

McCusker, J. et al. (2011). Prevalence and incidence of delirium in long-term care. International fournal of Geriatric Psychiatry, 26, 1152-1161.

Meagher, D., Adamis, D., Trzepacz, P. and Leonard, $M$. (2012). Features of subsyndromal and persistent delirium. British fournal of Psychiatry, 200, 37-44.

Ouimet, S. et al. (2007). Subsyndromal delirium in the ICU: evidence for a disease spectrum. Intensive Care Medicine, 33, 1007-1013.

Rockwood, K. (1993). The occurrence and duration of symptoms in elderly patients with delirium. Fournal of Gerontology, 48, M162-M166.

Siddiqi, N., House, A. O. and Holmes, J. D. (2006). Occurrence and outcome of delirium in medical in-patients: a systematic literature review. Age and Ageing, 35, 350-364.

Skrobik, Y. et al. (2010). Protocolized intensive care unit management of analgesia, sedation, and delirium improves analgesia and subsyndromal delirium rates. Anesthesia $\mathcal{E}$ Analgesia, 111, 451-463.

Tan, M. C. et al. (2008). Incidence and predictors of post-cardiotomy delirium. American fournal of Geriatric Psychiatry, 16, 575.

Trzepacz, P. T. et al. (2001). Validation of the Delirium Rating Scale-revised-98: comparison with the Delirium Rating Scale and the cognitive test for delirium. Fournal of Neuropsychiatry and Clinical Neurosciences, 13, 229-242.

Trzepacz, P. T. et al. (2012). Phenotype of subsyndromal delirium using pooled multicultural Delirium Rating Scale-Revised-98 data. Fournal of Psychosomatic Research, 73, 10-17.

von Gunten, A. and Mosimann, U. P. (2010). Delirium upon admission to Swiss nursing homes: a cross-sectional study. Swiss Medical Weekly, 140, 376-381.

Voyer, P., Richard, S., Doucet, L. and Carmichael, P. (2009). Detecting delirium and subsyndromal delirium using different diagnostic criteria among demented long-term care residents. Fournal of the American Medical Directors Association, 10, 181-188.

Witlox, J. et al. (2010). Delirium in elderly patients and the risk of postdischarge mortality, institutionalization, and dementia: a meta-analysis. $\mathcal{f} A M A, 304,443$.

World Health Organization (2010). International Classification of Diseases, 10th Revision (ICD-10). Available at: http://apps.who.int/classifications/icd10/browse/ 2010/en; last accessed 25 February 2013. 